\title{
Improved Outcomes With Standardized Convalescent Preterm Respiratory Care Practices
}

\author{
Michelle D Tyler, Neetu Singh, Matthew J McNally, Karen A Homa, and Alicia J Zbehlik
}

BACKGROUND: Chronic lung disease is the most common morbidity affecting very low birthweight (VLBW) infants. Many of these infants are discharged home on oxygen, placing significant emotional and financial burdens on families. We sought to reduce the proportion of VLBW infants requiring discharge home on oxygen by improving convalescent respiratory practices. METHODS: We performed a prospective quality-improvement project for infants with birth weights $\leq 1,500 \mathrm{~g}$ in a single neonatal ICU. Using Plan-Do-Study-Act cycles, we developed and implemented a room air challenge, oxygen reference chart, and a standardized oxygen delivery guideline. The primary outcome was the proportion of VLBW infants discharged home on oxygen. Secondary outcomes included rate of chronic lung disease and postmenstrual age when off all respiratory support. Balancing measures were postmenstrual age and weight at discharge, as well as unplanned readmissions. Statistical process control charts were used to monitor outcomes and balancing measures. RESULTS: The proportion of VLBW infants discharged home on oxygen decreased from $34.4 \%$ to $18.5 \%$ and $21.7 \%$ in the following two years $(P=.044$ and $P=.01$, respectively). G-Chart analysis showed a higher mean number of successes between failures. The rate of chronic lung disease decreased from $31.2 \%$ to $25.4 \%(P=.03)$. The mean postmenstrual age at discharge, mean weight at discharge, and readmission rate were unchanged. CONCLUSION: Standardization of convalescent respiratory care practices improved respiratory morbidities in VLBW infants. These interventions could be utilized in other NICUs with high incidence of respiratory morbidities despite improvement in delivery room practices. Key words: very low birthweight; premature infants; neonatal intensive care unit; bronchopulmonary dysplasia; chronic lung disease; oxygen inhalation therapy; quality improvement. [Respir Care 2019;64(9):1109-1115. (C 2019 Daedalus Enterprises]

\section{Introduction}

Chronic lung disease, defined as a physiologic requirement for supplemental oxygen at 36 weeks postmenstrual age (PMA), is the most common morbidity in premature infants. It affects approximately $40 \%$ of very low birthweight (VLBW) infants (infants with birth weights $\leq 1,500 \mathrm{~g}){ }^{1}$ Following discharge, infants with chronic lung disease grow poorly in all parameters: weight, length,

Dr Tyler presented a version of this paper at the American Academy of Pediatrics National Conference and Exhibition, held October 23, 2016, in San Francisco, California, and at the American Academy of Pediatrics National Conference and Exhibition, held September 15, 2017, in Chicago, Illinois.

The authors have disclosed no conflicts of interest.

Correspondence: Michelle D Tyler MD MPH, Children's Hospital at Dartmouth, Division of Neonatology, One Medical Center Drive, Lebanon, NH 03756. E-mail: michelle.d.tyler@hitchcock.org.

DOI: $10.4187 /$ respcare. 06684 
and head circumference. ${ }^{2}$ Chronic lung disease is independently associated with having worse long-term neurodevelopmental outcomes in later infancy and at school age. ${ }^{3,4}$ Infants requiring supplemental oxygen at discharge represent a subset of premature infants with severe chronic lung disease. This requirement leads to increased financial and psychological burdens on these families and additional health care costs to society..$^{5-7}$

In our level 3 neonatal ICU (NICU), the proportion of VLBW infants discharged home on oxygen historically has been high compared to the Vermont Oxford Network (VON), an international benchmark for NICUs. In 2014, of all VLBW infants discharged home, $34 \%$ required supplemental oxygen at discharge. Although an optimal rate is not known, this rate of discharge home on oxygen was markedly above the VON mean rate of $12 \%$ for the same year.

Reducing chronic lung disease rates in VLBW infants has been an area of emphasis for our NICU since 2009. Prior improvement efforts that were focused on resuscitation, stabilization, and reducing mechanical ventilation resulted in some success in reducing chronic lung disease. No significant efforts have focused on the later part of the hospital course during the convalescent phase of lung disease. Evidence shows that standardized oxygen delivery protocols and room air challenges can decrease instances of chronic lung disease..$^{8-11}$ We hypothesized that a quality-improvement initiative to standardize oxygen delivery protocols and room air challenges could reduce the rate of discharge home on oxygen and possibly reduce chronic lung disease rates for VLBW infants in our NICU. The specific aim of this quality-improvement effort was to reduce the proportion of VLBW infants discharged home on supplemental oxygen by $50 \%$ over 2 years.

\section{Methods}

\section{Context}

This quality-improvement project was conducted at a rural academic tertiary care hospital in a 30-bed level 3 NICU. There are $>350$ admissions annually, one third of which are outborn. There are approximately 70 VLBW infant admissions annually. The NICU is staffed by 5 neonatologists, 7 advanced providers (neonatal nurse practitioners or physician assistants), 3 neonatal fellows, 21 rotating pediatric residents, 25 respiratory therapists, and $>60$ neonatal nurses. Daily multi-disciplinary bedside rounds are conducted that help determine respiratory care plans. Oxygen saturation targets were 90-95\% for VLBW infants.

Both outborn and inborn VLBW infants $(<30$ weeks gestation and/or weight $\leq 1,500 \mathrm{~g}$ at birth) who were discharged home from our NICU were included. VLBW

\section{QUICK LOOK}

\section{Current knowledge}

Chronic lung disease is the most common morbidity effecting very low birthweight premature infants. This has persisted despite improvements in delivery room practices and early neonatal care.

\section{What this paper contributes to our knowledge}

Using quality-improvement methodology to implement a room air challenge, $\mathrm{F}_{\mathrm{IO}_{2}}$ table, and an oxygen delivery flow chart led to fewer very low birthweight infants requiring oxygen at discharge and fewer were diagnosed with chronic lung disease.

infants who died or were transferred to another hospital prior to discharge home were excluded.

This project was reviewed by the Dartmouth College Committee on the Protection of Human Subjects and was deemed exempt.

\section{Planning the Interventions}

An existing multidisciplinary team met twice monthly to identify areas for improvement in respiratory care practices in the NICU. This team consisted of champion physicians, nurse practitioners, nurses, respiratory therapists, care resource coordinators, and patient advocates. We nested our quality-improvement project within this group to complement the work already done to reduce chronic lung disease. Family members contributed through unstructured interviews. Using a microsystem approach, the team's first goal was to understand the current literature and the local problem. Key themes that emerged included variation in the oxygen-weaning process among providers, a lack of understanding of $\mathrm{F}_{\mathrm{IO}_{2}}$ when using different oxygen-delivery systems, and a desire for standardization of oxygen delivery.

Based on these findings, we began Plan-Do-Study-Act (PDSA) cycles and proceeded iteratively with the timeline of activities shown in Table 1. PDSA cycle 1 standardized a room air challenge on the basis of previous literature that reported improvements in outcomes with this intervention. ${ }^{8-10}$ Infants were eligible for a room air challenge if they were on supplemental oxygen without positive pressure and $\mathrm{F}_{\mathrm{IO}_{2}}$ was $<0.3$ at 34 weeks PMA. Oxygen amount and flows were weaned incrementally, and failure criteria followed the method described by Walsh et al. ${ }^{9}$ During the initial testing phase of this PDSA cycle, we found that the criteria for failure needed adjustment to include the oxygen saturation histogram data during the 30-min trial. By 
Table 1. Summary and Timeline of Improvement Activities

\begin{tabular}{|c|c|c|}
\hline Timeline & Dates & Description \\
\hline \multirow[t]{3}{*}{ Background work } & July to October 2015 & Review current literature \\
\hline & & Develop improvement team \\
\hline & & Begin unit education of the problem \\
\hline \multirow[t]{3}{*}{ Understand the problem } & October 2015 to February 2016 & Create fishbone diagram \\
\hline & & Create process map \\
\hline & & Interview key stakeholders \\
\hline \multirow[t]{5}{*}{ PDSA 1: Room air challenge } & February to May 2016 & Develop room air challenge protocol \\
\hline & & Begin test of change with room air challenge on small group of patients \\
\hline & & Modify protocol based on feedback \\
\hline & June to August 2016 & Implement room air challenge across unit \\
\hline & January to March 2017 & Train respiratory therapists on protocol \\
\hline \multirow[t]{4}{*}{ PDSA 2: Effective $\mathrm{F}_{\mathrm{IO}_{2}}$ table } & July to August 2016 & Create effective $\mathrm{F}_{\mathrm{IO}_{2}}$ reference table \\
\hline & & Test utility of table on small group of patients \\
\hline & & Modify based on nursing, respiratory therapist, parental feedback \\
\hline & October 2016 & Implement across unit by providing in all infants' bedside charts \\
\hline \multirow[t]{4}{*}{ PDSA 3: Oxygen delivery flow chart } & August to October 2016 & $\begin{array}{l}\text { Develop flow chart for oxygen delivery for very low birth weight infants } \\
\text { Obtain feedback from key stakeholders }\end{array}$ \\
\hline & October to December 2016 & Test with a small group of infants \\
\hline & January to March 2017 & Edit flow chart based on feedback \\
\hline & & Implement across unit \\
\hline
\end{tabular}

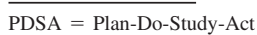

adding the failure criteria of the histogram showing $>10 \%$ of the $30-\mathrm{min}$ trial was with oxygen saturation $<90 \%$, we were able to account for the infants that had brief but frequent desaturations in room air. Respiratory therapists were trained on this protocol and monitored the room air challenges for all eligible infants. Our goal was to complete this room air challenge on all eligible infants before 36 weeks PMA.

PDSA cycle 2 was formulated based on concerns from staff and parents about changes to $\mathrm{F}_{\mathrm{IO}_{2}}$ with different oxygen-delivery methods. An effective $\mathrm{F}_{\mathrm{IO}_{2}}$ table was created for different infant weights and oxygen delivery methods based on published literature. ${ }^{12,13}$

PDSA cycle 3 addressed the issue of variation in oxygen-delivery method. After review of literature and key stakeholder discussions, the team developed an oxygendelivery flow chart to standardize the method of oxygen delivery on the basis of the infant's PMA (Fig. 1). This was a guideline for providers and staff to follow with 3 distinct parts in the VLBW convalescent phase of their course. The first part was focused on providing appropriate airway pressure by keeping VLBW infants $<34$ weeks PMA on nasal CPAP until ready for room air. The second part of the flow chart focused on utilizing developmentally appropriate oral feeding cues, using $1 / 4 \mathrm{~L} / \mathrm{min}$ nasal cannula blended oxygen if low $\mathrm{F}_{\mathrm{IO}_{2}}$ support was needed. If an infant could not pass a room air challenge by 36 weeks PMA, he was then placed on $1 / 8 \mathrm{~L} / \mathrm{min}$ nasal cannula unblended oxygen. This third part of the flow chart al- lowed the team to focus care toward discharge planning. This flow chart was tested on a small group of patients and modified on the basis of provider and staff comments.

\section{Study of the Interventions}

The improvement team met twice monthly throughout the project to evaluate the interventions, staff and nursing responses to the interventions, as well as any unanticipated effects. Outcome and balancing measures were tracked regularly and discussed with the team. Real-time chart review was used to monitor for VLBW infants eligible for the interventions.

\section{Measures}

The primary outcome measure was the proportion of VLBW infants discharged home on oxygen. The secondary outcome was the proportion of VLBW infants diagnosed with chronic lung disease, as defined by a supplemental oxygen requirement at 36 weeks PMA. To measure progress, we included a process measure of average PMA for VLBW infants to discontinue respiratory support. Balancing measures included PMA at discharge, weight at discharge (to ensure that growth was not affected by discontinuing oxygen too early), and unplanned readmissions within $30 \mathrm{~d}$. 


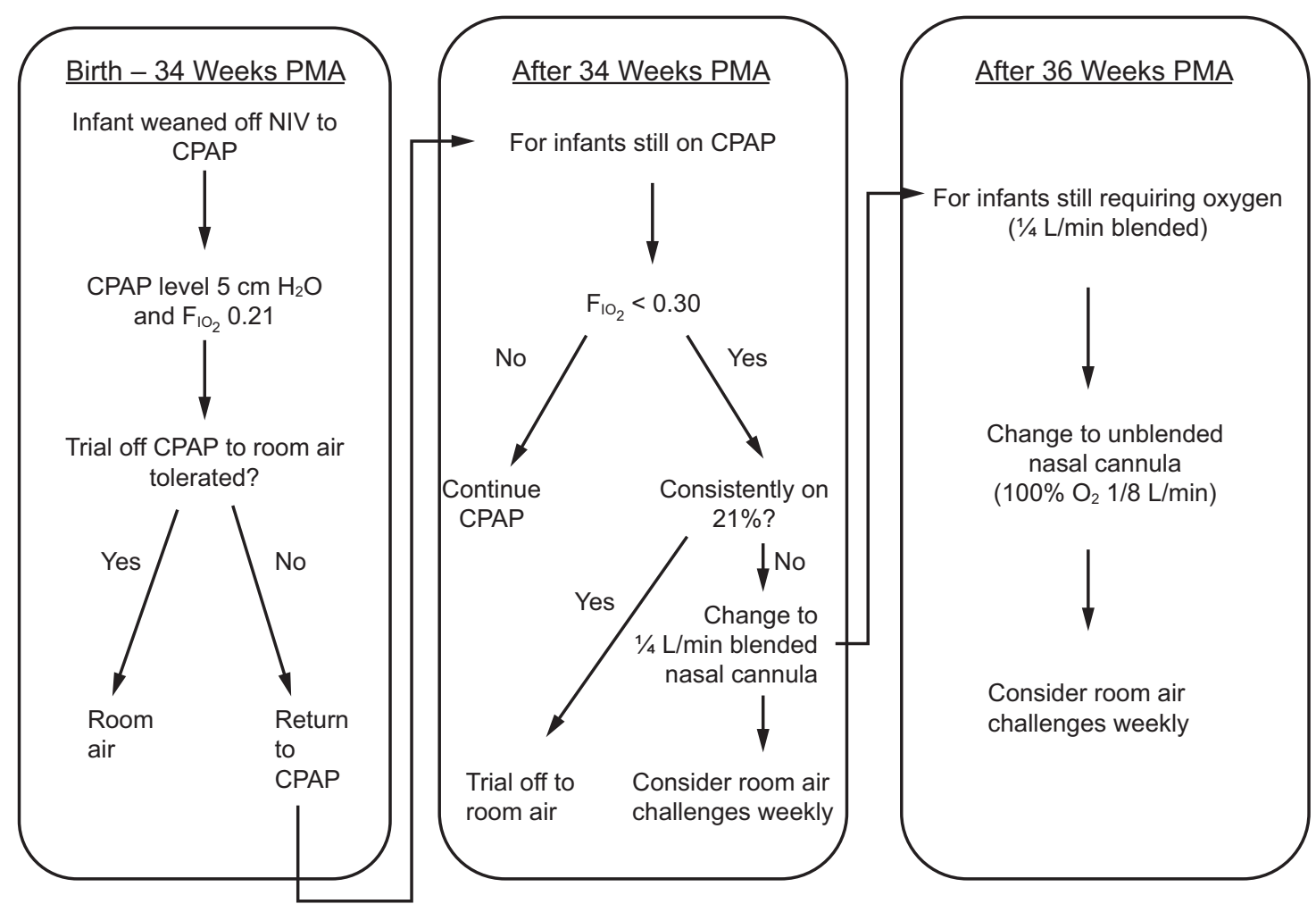

Fig. 1. Very low birthweight oxygen-delivery flow chart developed in the Plan-Do-Study-Act cycle 3. PMA = postmenstrual age.

Table 2. Comparison of Subject Characteristics for the Baseline Year (2014) and the Intervention Years (2015 and 2016)

\begin{tabular}{|c|c|c|c|c|c|}
\hline & 2014 & 2015 & 2016 & $P(2014$ vs 2015$)$ & $P(2014$ vs 2016$)$ \\
\hline Gestational age, weeks & $275 / 7 \pm 2.7$ & $28 \pm 2.4$ & $28 \pm 2.6$ & .60 & .55 \\
\hline Birth weight, $\mathrm{g}$ & $1041 \pm 306$ & $1064 \pm 297$ & $1048 \pm 266$ & .68 & .89 \\
\hline Male & $29(46)$ & $30(56)$ & $43(61)$ & .20 & .006 \\
\hline Antenatal steroids & $55(87)$ & $46(88)$ & $58(84)$ & .07 & .39 \\
\hline Inborn & $57(90)$ & $49(92)$ & $55(78)$ & .10 & .36 \\
\hline White race & $57(90)$ & $48(91)$ & $61(87)$ & .08 & .30 \\
\hline Multiple birth & $16(25)$ & $11(21)$ & $11(16)$ & .08 & .13 \\
\hline
\end{tabular}

\section{Analysis}

The proportion of VLBW infants discharged home on oxygen, incidence of chronic lung disease, and weight at discharge were all evaluated annually and compared to VON means and first and third quartiles. Statistical process control charts were used to monitor the remaining measures, with exception of readmissions, and the charts were evaluated for any special-cause variations that would indicate intervention-related changes. Because discharge home on oxygen was a rare event, a G-chart was used to track in real time the number of VLBW infants discharged home without oxygen (suc- cesses) between infants discharged with oxygen (failures). Published special-cause variation rules were used. ${ }^{14}$ Demographic and clinical characteristics for the infants were tracked. A 2-sample Student $t$ test (2-sided) was used to compare differences in means for continuous variables, and the chi-square test was used to compare categorical variables.

\section{Results}

From January 2014 through December 2016, 186 VLBW infants were admitted to the NICU: 63 in the baseline year, and 53 and 70 in the following intervention years. Demo- 


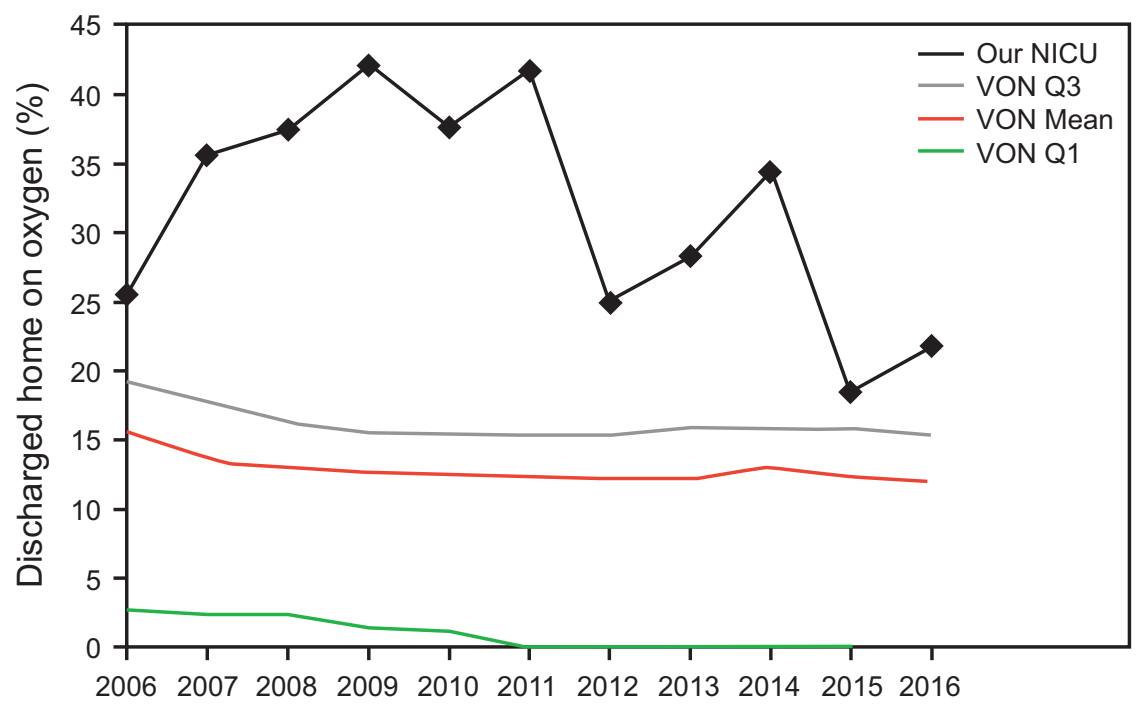

Fig. 2. The proportion of very low birthweight infants in our neonatal ICU discharged home on oxygen per year compared to the Vermont Oxford Network (VON) mean, 3rd quartile (VON Q3), and 1st quartile (VON Q1).

graphic data were similar between the 3 years, with the exception of significantly higher percentage of males in 2016 (Table 2).

Of the infants admitted, 27 were discharged to home in 2015 and 46 in 2016, and the remaining were transferred to a local hospital (16 in 2015 and 19 in 2016) or died before discharge. Five of the 27 infants (18.5\%) in 2015 and 10 of the $46(21.7 \%)$ in 2016 required oxygen at discharge, as shown in Figure 2. Both of these rates were significantly lower compared to the baseline of $34.4 \%$ ( $P=.044$ for $2015, P=.01$ for 2016), although both rates remained above both the VON mean of $12 \%$ and the third quartile of $15 \%$. Rates of chronic lung disease also decreased from $31.2 \%$ in 2014 to $25.0 \%$ and $25.4 \%$ in 2015 and 2016, respectively ( $P=.08$ and .03 , respectively). This was just above the VON chronic lung disease mean of $24 \%$ and below the third quartile of $29 \%$.

Baseline data of discharges tracked using a G-chart demonstrated a mean number of infants successfully discharged home without oxygen between failures was 1.79 with an upper control limit of 8.48 (Fig. 3). One special-cause signal, a point above the upper control limit, was noted during the baseline period. This indicated that the system was not in control at baseline. After starting the project, the mean increased to 3.6 and the upper control limit increased to 15.81 , and there were no special-cause signals. The timing of the interventions is noted in Figure 3.

Regarding other measures, there were no special-cause signals noted for PMA at discontinuation of respiratory support. One special-cause signal was noted for PMA at discharge with a point above the upper control limit in 2016, but no further signals were seen and the data re- turned to baseline (data not shown). Mean weight at discharge (2,608 $\mathrm{g}$ in 2015 and 2,823 $\mathrm{g}$ in 2016) continued to be above the VON mean $(2,255 \mathrm{~g})$. There was no difference in rate of unplanned readmissions.

\section{Discussion}

We describe a successful quality-improvement initiative in which standardization of room air challenges and oxygen delivery to VLBW infants was associated with nearly halving the proportion of infants who were discharged home on oxygen. Rates of chronic lung disease for all VLBW infants also decreased by $>30 \%$ during the same time. Despite these improvements, we remained above the VON third quartile for infants discharged home on oxygen. There were no perceived effects of the interventions on PMA and weight at discharge, indicating that discharges were not delayed and nutritional status was not affected with these interventions. Before starting this work, there was notable variation in our NICU regarding which respiratory support delivery system to use and when to trial off support. These variations appeared to be provider-dependent and not based on the physiology of the infant. By standardizing these processes, we were able to engage providers, staff, and family in reducing the variability and improving outcomes for the infants.

This project was unique in its focus on room air challenges and oxygen delivery in the convalescent phase of lung disease in premature infants. Walsh and colleagues 9,10 previously reported that using the physiologic definition of performing a time-specific and controlled room air challenge could decrease incidence of chronic lung disease and 


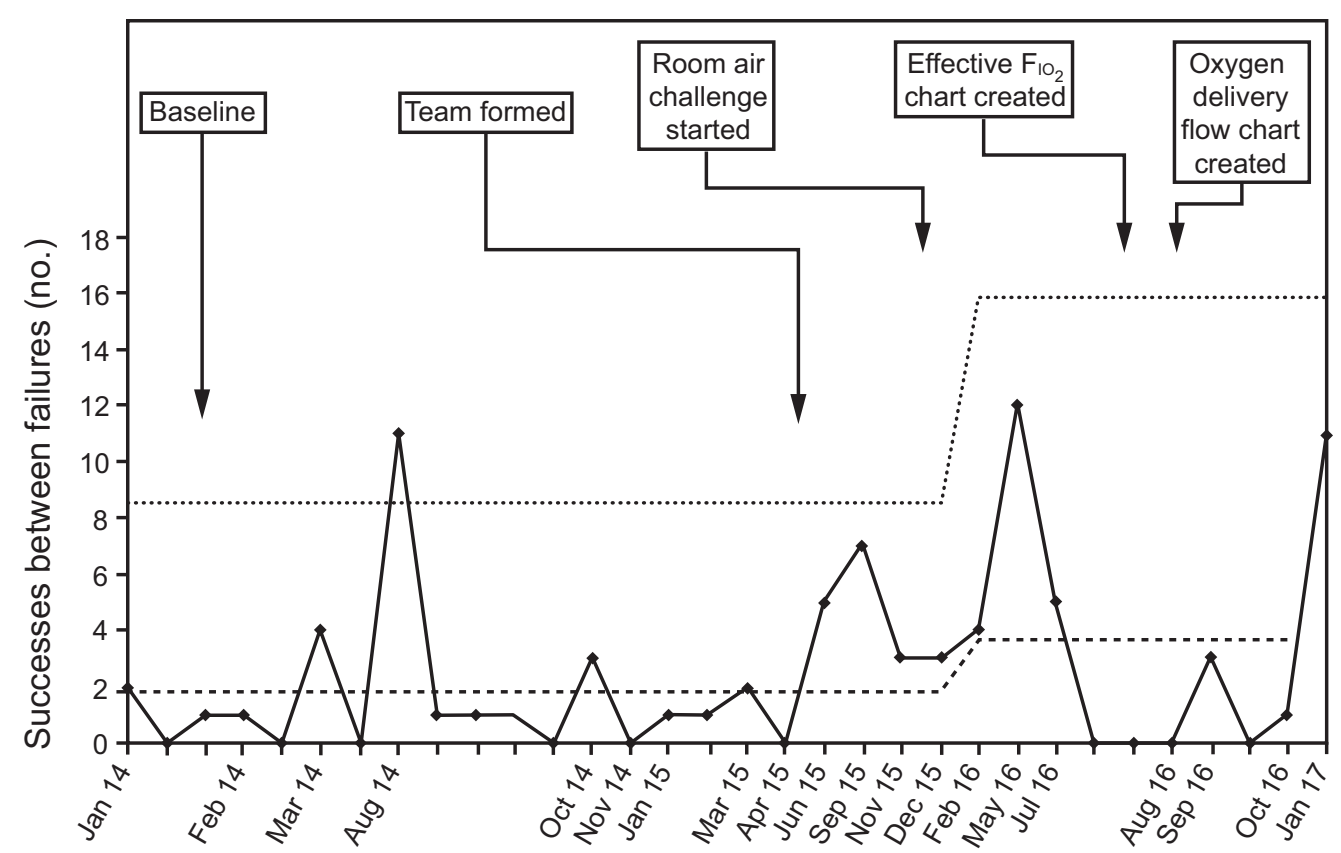

Fig. 3. The number of very low birthweight infants discharged home not requiring oxygen (ie, successes) between infants discharged home requiring oxygen (failures) from January 2014 to January 2017. The upper control limit (dotted line) and mean (dashed line) are shown for baseline and during the project.

reduce variations in the diagnosis. Although many institutions have accepted this definition, our unit had not started this practice, nor is there much literature reporting on the implementation and sustainment of this practice in individual NICUs.

Previously published quality-improvement work on reducing respiratory morbidities has largely focused on the initial stabilization and respiratory care practices of VLBW infants. Single-center and large, multi-center collaborations have implemented potentially better practices, including vitamin A, postextubation and delivery room nasal CPAP, reduction in ventilator days, and permissive hypercarbia. ${ }^{15-21}$ Results from these efforts are mixed, with some showing significant reductions in chronic lung disease and others finding limited success. Only some of these reported on discharge home on oxygen, and few implemented changes during the convalescent phase. Although we had successfully implemented similar evidence-based and potentially better practices, including initial stabilization on CPAP, early extubation to noninvasive respiratory support after INSURE (INtubate-SURfactant-Extubation), and medication for chronic lung disease prophylaxis (caffeine and vitamin A), we did not see significant changes in chronic lung disease rate or in the rate of discharge home on oxygen. Ours is one the few studies reporting oxygenweaning strategies during the convalescence period to affect rate of discharge home on oxygen and chronic lung disease.

\section{Limitations}

This quality-improvement project took place in a relatively small unit with a small number of neonatologists and associate providers. This likely led to an easier consensus regarding interventions; this approach may be more challenging in units with a higher census and larger staff. Also, our unit has participated in other quality initiatives, both locally and through VON. This culture likely affected staff attitudes, making them more willing to accept change, and this might limit the generalizability of the interventions. Due to the small number of qualifying infants and the relative rarity of the outcome, we made comparisons to annual VON data to assist our analysis of the main outcome. This approach made real-time tracking difficult. We attempted to mitigate this by using a G-chart to measure elapsed time between undesired occurrences.

It is clear that our unit was an outlier, although it is not completely clear why. Although we saw improvement in our rate of discharge home on oxygen, it remains above the VON average and third quartile. The cause for this is not immediately known because we do not believe that our population is significantly more ill than other NICUs. We are continuing to work to improve our practices and optimize care for these infants. It is also possible that, with the degree to which our rate was above the mean, some of the improvement could be due to regression to the mean. This had not happened in the previous 10 years, however, de- 
spite previously mentioned improvements in our respiratory care practices.

\section{Conclusions}

We describe a successful quality-improvement effort to decrease discharge home on oxygen and chronic lung disease through implementation of standardized room air challenges, oxygen delivery, and weaning practices in the convalescent phase of lung disease in preterm infants. Acceptance of these interventions in other institutions could lead to reduced variation in oxygen administration, decreased respiratory morbidities, and avoid the emotional and financial stress of discharging a preterm infant home with oxygen.

\section{ACKNOWLEDGMENTS}

The authors would like to thank the nurses, respiratory therapists, associate providers, and neonatologists for assistance and appreciation for this work. We would also like to thank the Leadership Preventive Medicine Residency program and faculty for their intellectual support through this project and reviewing the manuscript.

\section{REFERENCES}

1. Stoll BJ, Hansen NI, Bell EF, Walsh MC, Carlo WA, Shankaran S, et al. Trends in care practices, morbidity, and mortality of extremely preterm neonates, 1993-2012. JAMA 2015;314(10):1039-1051.

2. Natarajan G, Pappas A, Shankaran S, Kendrick DE, Das A, Higgins $\mathrm{RD}$, et al. Outcomes of extremely low birth weight infants with bronchopulmonary dysplasia: impact of the physiologic definition. Early Hum Dev 2012;88(7):509-515.

3. Hoekstra RE, Ferrara TB, Couser RJ, Payne NR, Connett JE. Survival and long-term neurodevelopmental outcome of extremely premature infants born at 23-26 weeks' gestational age at a tertiary center. Pediatrics 2004;113(1):e1-e6.

4. Short EJ, Klein NK, Lewis BA, Fulton S, Eisengart S, Kercsmar C, et al. Cognitive and academic consequences of bronchopulmonary dysplasia and very low birth weight: 8-year-old outcomes. Pediatrics 2003;112(5):e359.

5. McLean A, Townsend A, Clark J, Sawyer M, Baghurst P, Haslam R, et al. Quality of life of mothers and families caring for preterm infants requiring home oxygen therapy: a brief report. J Paediatr Child Health 2000;36(5):440-444.

6. Singer LT, Salvator A, Guo S, Collin M, Lilien L, Baley J. Maternal psychological distress and parenting stress after the birth of a very low-birth-weight infant. JAMA 1999;281(9):799-805.
7. Behrman RE, Butler AS. A Systematic Review of Costs Associated with Preterm Birth. Preterm Birth: Causes, Consequences, and Prevention. Washington (DC): National Academies Press (US); 2007.

8. Walsh M, Engle W, Laptook A, Kazzi SNJ, Buchter S, Rasmussen $\mathrm{M}$, et al. Oxygen delivery through nasal cannulae to preterm infants: can practice be improved? Pediatrics 2005;116(4):857-861.

9. Walsh MC, Wilson-Costello D, Zadell A, Newman N, Fanaroff A. Safety, reliability, and validity of a physiologic definition of bronchopulmonary dysplasia. J Perinatol 2003;23(6):451-456.

10. Walsh MC, Yao Q, Gettner P, Hale E, Collins M, Hensman A, et al Impact of a physiologic definition on bronchopulmonary dysplasia rates. Pediatrics 2004;114(5):1305-1311.

11. Jackson JK, Ford SP, Meinert KA, Leick-Rude MK, Anderson B, Sheehan MB, et al. Standardizing nasal cannula oxygen administration in the neonatal intensive care unit. Pediatrics 118(Suppl 2): S187-S196, 2006.

12. Finer NN, Bates R, Tomat P. Low flow oxygen delivery via nasal cannula to neonates. Pediatr Pulmonol 1996;21(1):48-51.

13. Group S-RMS. Supplemental therapeutic oxygen for prethreshold retinopathy of prematurity (STOP-ROP), a randomized, controlled trial. I: primary outcomes. Pediatrics 2000;105(2):295-310.

14. Carey RG, Stake LV. Improving healthcare with control charts: basic and advanced SPC methods and case studies. Milwaukee, WI: ASQ Quality Press; 2003.

15. Sharek PJ, Baker R, Litman F, Kaempf J, Burch K, Schwarz E, et al. Evaluation and development of potentially better practices to prevent chronic lung disease and reduce lung injury in neonates. Pediatrics 2003;111(Suppl E1):e426-e431.

16. Payne NR, LaCorte M, Karna P, Chen S, Finkelstein M, Goldsmith JP, et al. Reduction of bronchopulmonary dysplasia after participation in the breathsavers group of the Vermont Oxford network neonatal intensive care quality improvement collaborative. Pediatrics 2006;118(Suppl 2):S73-S77.

17. Birenbaum H, Pfoh E, Helou S, Pane M, Marinkovich G, Dentry A, et al. Chronic lung disease in very low birth weight infants: persistence and improvement of a quality improvement process in a tertiary level neonatal intensive care unit. J Neonatal Perinatal Med 2016;9(2):187-194.

18. Birenbaum HJ, Dentry A, Cirelli J, Helou S, Pane MA, Starr K, et al Reduction in the incidence of chronic lung disease in very low birth weight infants: results of a quality improvement process in a tertiary level neonatal intensive care unit. Pediatrics 2009;123(1):44-50.

19. Payne NR, Finkelstein MJ, Liu M, Kaempf JW, Sharek PJ, Olsen S. NICU practices and outcomes associated with 9 years of quality improvement collaboratives. Pediatrics 2010;125(3):437-446.

20. Lapcharoensap W, Bennett M, Powers R, Finer N, Halamek L, Gould $\mathrm{J}$, et al. Effects of delivery room quality improvement on premature infant outcomes. J Perinatol 2017;37(4):349.

21. Walsh M, Laptook A, Kazzi SN, Engle WA, Yao Q, Rasmussen M, et al. A cluster-randomized trial of benchmarking and multimodal quality improvement to improve rates of survival free of bronchopulmonary dysplasia for infants with birth weights of less than 1,250 grams. Pediatrics 2007;119(5):876-890. 